\title{
Study on the Effect of Grassroots Community Services on Disabled Elderly Care System
}

\author{
Hongling $\operatorname{Pan}^{1}$ \\ ${ }^{1}$ Luohe Medical College, Luohe, Henan, 462000 \\ ${ }^{a}$ email
}

Keywords: Community Health Services; Urban; Long-Term Care; Disabled Elderly

\begin{abstract}
Analysis of long-term care for the elderly people can lose the status quo, field research in elderly care hospital in Henan Province, Zhengzhou Erqi apartments for the elderly and community health services involved in Luohe disabled elderly long-term care model in which community health services mode of long-term care, community health service station stationed pension agency model, social home care model, discuss community health services long-term care system in the role in urban disabled elderly; proposes should accelerate China's national conditions of long-term care service system construction, promote the combination of community health services long-term care service system, the establishment of the basic framework..
\end{abstract}

\section{Introduction}

Long-term care generally refers to life can not take care of themselves and must rely on others to take care of elderly disability provide integrated services, there is no single definition international. Taiwan scholars believe that long-term care refers to a long-term care service life due to suffering from incapacitating leading to life can not take care of the elderly carried out, including activities of daily assistance, psychological comfort, support the economy, but generally refers to 6 months of service. In Western countries, long-term care service system is an important system arrangement of its public services. And in long-term care, involving the most is health care, rehabilitation care content, it is also above two service resources are relatively scarce, restricted the development of long-term care industry. At present, China vigorously promote community health services are preventive, medical care, rehabilitation, health education and family planning services as a whole, is a safe, effective, convenient and economical basic health services. So the study of community health services in long-term care services for disabled elderly in the role, it is very urgent and necessary. The issue of long-term care in the country for the first time municipal districts within the region disabled elderly population census, in order to grasp the basic situation of the population, while select urban elderly long-term care work carried out in Henan good, representative Erqi District, Luohe Yuan Huiqu, field research, thematic seminars and in-depth interviews, community health services in the city of disabled elderly long-term care system in effect were discussed.

\section{Long-Term Care for the Elderly People can Lose the Status}

Zhengzhou City, select a representative Erqi Old Town, the status of the city urban communities all over the age of 60 need long-term care services for 836 disabled elderly of baseline surveys, results were as follows.

Long-term care for disabled elderly living conditions aged 80 to 89 years age group, followed by 70 years to 79 years of age; disabled elderly divorce rate is $2.63 \%$; disabled elderly educational level is not high; Alone ratio than high of $33.97 \%$, and the age, long-term care for disabled elderly living alone increased the proportion; $72.63 \%$ of the disabled elderly mental confidant spouses and children; $70.69 \%$ of the disabled elderly by their own support; $66.31 \%$ of the disabled elderly I think health care costs is a major economic costs in addition to daily life.

Long-term care for disabled elderly health and life satisfaction of disabled elderly life satisfaction is not high, / 0 and satisfied / very satisfied accounted for only $12.90 \%$, "General" and accounted for 50.54\%; health satisfaction than lower life satisfaction, satisfied and very satisfied 
with only $1.09 \%, 52.17 \%$ are not satisfied; $95.93 \%$ of the disabled elderly suffering from various chronic diseases, which in turn have a higher prevalence of hypertension (59.09\% ), coronary heart disease (31.10\%), diabetes (19.50\%), bone and joint diseases (19.50\%).

Long-term disability care service needs of the elderly can not take care of themselves first recourse for their children, followed by spouses and nannies; the main reason is that the disease disability, injury, old age; $86.32 \%$ of the disabled elderly want to live at home; when long-term hospitalization $57.61 \%$ of the disabled elderly want to take care of by their families; $58.95 \%$ of the disabled elderly can afford long-term care represents a cost of 500 yuan per month; $94.74 \%$ of the disabled elderly with all kinds of medical care; disabled elderly hope community demand for services provided by the main home

Chamber Rehabilitation (65.16\%), regular visits (61.05\%), help Pharmaceuticals (60.00\%), home care (52.68\%), health consultation (50.53\%), but community service satisfaction is only 23.16 percent.

\section{Disabled Elderly Long-Term Care Model of Community Health Service Intervention}

Our group of Henan elderly care hospitals, elderly apartments and Erqi Luohe Yuan Huiqu conduct field research, thematic seminars and in-depth interviews, disabled elderly these types of models work under long-term care, are very unique, also well suited to local conditions, so the local government and the people's approval.

Long-Term Care Community Health Service mode is Community Health Center organized long-term care ward. Our group Henan elderly care hospital survey, is based on Erqi gongchenqiao center built on long-term care services to the elderly featuring hospital. Hospital construction area of 10,000 square meters, a total of nearly 400 elderly care beds. Since 15 years, it has treated more than 20,000 cases of elderly patients, about two-thirds of long-term care patients. Mode of running the hospitals hospital, quality of service, and other characteristics, in the province and the country has a certain influence.

Long-term care community health services mode (mode elderly care hospital) is an ideal city mode disabled elderly long-term care, long term care services for the urban elderly has played an exemplary role. According to the Ministry of Health "urban community health services management approach": community health service centers in principle inpatient beds, inpatient beds should be turned to existing care and rehabilitation beds as the main function, or be revoked. Currently most of the urban community health service centers from the original primary, secondary conversion from the hospital, there are still a considerable part has some beds. Therefore, in order to rely on community health service centers, the creation of elderly long-term care beds, easy to carry out in coordination with the regional health planning, conservation of health resources. Community health services can be within a certain range for the disabled elderly to provide medical care to the elderly disease care, long term care services mainly distinguished from the pension-based old-age benefits to medical institutions and hospital-based elderly.

Community Health Service Stations stationed pension agency model Gongshu old apartment was invested by Erqi government, state-owned pension agency Erqi Civil Administration direct management, a total of 215 beds, the occupancy rate of 99\%. That initial period of cooperation by the Gongshu Xiangfu town community health service centers and apartments for the elderly, the elderly apartment business houses, community health centers sent four medical personnel assigned to provide outpatient medical and long-term care services. Since the start of cooperation in October 2007, has been an apartment complex, check praise for the elderly and their families, get a good social and economic benefits. Check-in services for the elderly community health service stations provide very pleased that the clinic convenient, inexpensive cost, can basically solve their pain, lived at ease; and dispensing doctor elderly family members think is very convenient, simplifying the large hospital complex treatment processes and procedures, reducing their burden to make them better able to plunge into their work, and therefore are very satisfied; as the old apartment and the Civil Administration, it does not need to because the additional staffing and equipment costs can be reduced accordingly spending, and community health services can be settled in an apartment 
complex to attract more older people to stay and increase economic efficiency; for community service station, the old apartments business premises, do not need to lease additional station house, community service center this part can reduce expenses, but also taking into account the medical community, to facilitate people around treatment, without duplication of investment personnel and equipment. Combined Xiangfu Town Community Health Service Center of Chinese medicine characteristics and the actual situation of the elderly apartment in 2010 will increase in literacy elderly Chinese medicine, Chinese medicine and promote appropriate technology, and consider providing counseling services to enable the elderly have physiological stay and mental health.

Socialization home care model Luohe Yuan Huiqu existing 24,000 over the age of 60, 17.1\% of the total population. Among them, the empty nest elderly people living alone has 0.6 million people, accounting for $47.9 \%$ of the total elderly. Yuan Huiqu is the center of the city of Luohe, machine

Insufficient supply configuration pension, family pension function is increasingly weakened. Beginning in 2010, Yuan Huiqu actively build new social home care service system, after four years of practice, the practice of Yuan Huiqu won the society, especially the elderly welcome. 2008 Luohe Yuan Huiqu "The government purchase home care services," won the Fourth China Local Government Innovation Award winner. Luohe Yuan Huiqu socialization home care model is based on family-centered, community-based, elderly day care, life care, housekeeping

Spirit of service and comfort as the main content, above the door, and community day-care services as the main form, and the introduction of the pension agency specialized services way home care service system. According to the characteristics of the disabled elderly live, Luohe Yuan Huiqu using a wide range of community health services

Service agency resources to provide low-cost health care to address the medical needs of the elderly disability: 2013, Yuan Huiqu to more than 20,070 years of age or older issuing "Yuan Huiqu community basic medical services card", these old people in area belong to seven community health service centers and 24 service stations to enjoy low-cost medical care. They can enjoy preferential services are mainly three: pharmaceuticals, medical devices only charge a one-time cost; technical services rendered free of medical personnel; other medical expenses tickets. The low-income groups in the basic medical community

After the service system was introduced, per capita health care costs 28.16 yuan, 80 yuan more than the region's average cost 65\% lower. Low-cost health service coverage from 2008 to all 60 years of age or older. Luohe Yuan Huiqu "low health" is not only the form of a system to help the elderly, so that the elderly can also choose the hospital, this approach is the first in Henan Province. Luohe Yuan Huiqu mode take advantage of the government, society, the power of individuals to serve disabled elderly services, there is a very good pension system in the future, combining community health service system, long-term care of the disabled elderly problem will be solved, the sustainable development model is to be expected.

\section{Policy Recommendations}

suitable for China to speed up the long-term care service system construction of the long-term care services due to the lack of serious, long-term care problem is very urgent reasons to build suitable for China's long-term care service system is imminent. Primarily include: the establishment of appropriate services, management and oversight bodies, responsible for overall planning and guidance of long-term care services for the cause of development; to carry out long-term care business insurance under the government support as soon as possible; explore the establishment of long-term care social insurance; encourage social forces to energetically initiate is old social service agencies for the elderly disability specialization and standardization of long-term care services.

The Government should introduce policies to promote the establishment of community health services and the combination of long-term care service system to improve the basic framework of long-term care services help to improve the overall health care services, especially the efficiency and effectiveness of community health service system, and long-term care system development is inseparable from the health services, especially the strong support of community health services, the two are complementary and inseparable. 
Development and Community Health Service in close connection with the community long-term care service system for community long-term care services will become long-term care service system in the core content. Community Health Service Intervention disabled elderly long-term care has the advantage of community health services to the community as a platform to provide systems, continuous, similar to the familiar neighborhood formed between the integrated health services and community physicians. Because community health services are for common disease, common disease treatment and routine health maintenance. Therefore, consumers have a certain ability to select, you can well overcome the problem of high consumption of medical health care market monopoly and physician-induced demand brought about. Community Health Services is to provide medical services in the service object "house" for which the mobility of the elderly have a strong appeal.

Non-formal long-term care services is the basis of long-term care service system

Over a long period of time, family members will be one of the main providers of long-term care of the elderly. Community health services in health education and medical support for the disabled elderly family members, there would be able to play a greater role.

Type of long-term care institutions throughout the long-term care services is a powerful backup system as small and gradually increases the education level of the family structure, more and more older people will accept the type of long-term institutional care institutions. With the enhancements to improve the socio-demographic and cultural qualities and ability to pay, with preventive health care services for development agencies based long-term care services will be very great. Community Health Service and the combination of long-term care institutions based services will be one of the best model for future long-term care of the disabled elderly.

\section{Acknowledgements}

This is the Science and Technology Department of Henan Province soft science research project planning fiscal 2015. Item Number: 152400410368

\section{References}

[1] Song Shenchao, Huang Chung elderly doctor CAUSE panel discussions investigation [J]. Chinese Health Service Management, 2000 (141): 176-177.

[2] Xie Meie elderly long-term care related topics [M]. Taipei: Laurel Books Ltd., 1993: 14-26.

[3] Liu Hongxia. status and progress of community nursing in China [J]. Nursing Research, 2004,18 (4B):. 1413-1415.

[4] Weng Lifang. challenge the status quo and the development of community nursing in China face [J]. Nursing Research, 2004,18 (1A): 17. 